\title{
Coagulative biomarkers on admission to the ICU predict acute kidney injury and mortality in patients with septic shock caused by intra-abdominal infection
}

This article was published in the following Dove Press journal:

Infection and Drug Resistance

\author{
Zhipeng $\mathrm{Xu}{ }^{\prime}$ \\ Baoli Cheng' \\ Shuiqiao $\mathrm{Fu}^{2}$ \\ $\mathrm{Xu} \mathrm{\textrm {Liu } ^ { 3 }}$ \\ Guohao Xie' \\ Zhongwang $\mathrm{Li}^{1}$ \\ Yun $\mathrm{ji}^{2}$ \\ Qinghui $\mathrm{Fu}^{2}$ \\ Zhinan $\mathrm{Xu}\left(\mathbb{D}^{4}\right.$ \\ Xiangming Fang' \\ 'Department of Anesthesiology and \\ Intensive Care Medicine, The First \\ Affiliated Hospital, School of Medicine, \\ Zhejiang University, Hangzhou City, \\ Zhejiang Province, People's Republic of \\ China; ${ }^{2}$ Department of Surgical Intensive \\ Care Unit, The Second Affiliated \\ Hospital, School of Medicine, Zhejiang \\ University, Hangzhou City, Zhejiang \\ Province, People's Republic of China; \\ ${ }^{3}$ Department of Intensive Care Unit, The \\ Affiliated Hospital of Guizhou Medical \\ University, Guiyang City, Guizhou \\ Province, People's Republic of China; \\ ${ }^{4}$ Institute of Biological Engineering, \\ College of Chemical and Biological \\ Engineering, Zhejiang University, \\ Hangzhou City, Zhejiang Province, \\ People's Republic of China
}

Correspondence: Xiangming Fang Department of Anesthesiology and Intensive Care Medicine, The First Affiliated Hospital, School of Medicine, Zhejiang University, 79 Qingchun Road, Hangzhou City, Zhejiang Province 310003, People's Republic of China $\mathrm{Tel} / \mathrm{Fax}+8657188208006$

Email xmfang@zju.edu.cn
Purpose: Sepsis-associated coagulopathy (SAC) contributes to the development of multiple organ failure (MOF) and increasing mortality. The present study was conducted to investigate whether coagulative biomarkers on admission to the intensive care unit (ICU) can predict acute kidney injury (AKI) and mortality in patients with septic shock caused by intra-abdominal infection (IAI).

Patients and methods: An observational retrospective study was conducted in the surgical ICU. We studied patients who met the criteria of septic shock (Sepsis-3) caused by IAI between January 1, 2013, and December 31, 2016. By adjusting for baseline characteristics, multivariate regression analyses were employed to identify independent risk factors for predicting AKI and mortality.

Results: Of the 138 enrolled patients, 65 patients developed AKI. The patients who developed AKI exhibited a dramatically higher Sequential Organ Failure Assessment (SOFA) score (median, 12), Acute Physiology and Chronic Health Evaluation (APACHE) II score (median, 27.5) and mortality rate. In both models, we found that activated partial thromboplastin time (APTT) (odds ratio $(\mathrm{OR})=1.074,95 \%$ confidence interval (CI) 1.030 $1.120, p=0.001)$, prothrombin time $(\mathrm{PT})(\mathrm{OR}=1.162,95 \% \mathrm{CI} 1.037-1.302, p=0.010)$ and $\mathrm{D}-$ dimer level $(\mathrm{OR}=1.098,95 \%$ CI $1.002-1.202, p=0.045)$ on admission to the ICU were significant risk factors for AKI. Moreover, Cox regression analysis showed that prolonged APTT $(\mathrm{OR}=1.065,95 \%$ CI $1.025-1.107, p=0.001)$ was independently associated with high mortality.

Conclusion: In patients with septic shock caused by IAI, APTT, PT and D-dimer level on admission to the ICU were significantly associated with AKI. Furthermore, APTT was an independent predictor of 30-day mortality.

Keywords: coagulopathy, biomarker, septic shock, acute kidney injury, intra-abdominal infection, mortality

\section{Introduction}

Septic shock is a serious response to infection that involves underlying circulatory and cellular metabolic abnormalities and has a substantial effect on health resources and mortality. ${ }^{1}$ Although the guidance of Surviving Sepsis Campaign bundles has led to a decline in incidence, septic shock continues to account for $62 \%$ of overall shock cases, with hospital mortality rates greater than $40 \% .^{1-3}$ In the surgical intensive care unit (ICU), intra-abdominal infection (IAI) is the most common cause of sepsis, resulting in an overall mortality rate of $10.5 \%$ worldwide. $^{4,5}$ 
Although hospital mortality related to septic shock has declined, the rate of organ dysfunction remains high. Acute kidney injury (AKI) is one of the most frequent complications of sepsis, which endangers $30-40 \%$ of patients in the ICU. ${ }^{6}$ More than $45 \%$ of patients with septic shock suffer from AKI, and all of them may display poor outcomes, including longer ICU and hospital stays, chronic kidney disease (CKD), end-stage renal disease (ESRD) and increased mortality. ${ }^{7-9}$ However, thus far, there have been no reliable biomarkers that could predict AKI following sepsis. In addition, $>50 \%$ loss of renal function is required to induce changes in $\mathrm{SCr}$, limiting the ability to provide early intervention for AKI in septic patients. ${ }^{10,11}$ Therefore, the need to develop more sensitive biomarkers for predicting $\mathrm{AKI}$ and guiding further supporting treatment to reduce the exacerbation of renal function is urgent.

Mounting evidence indicates that sepsis-associated coagulopathy (SAC), which causes microvascular thrombosis and subsequent multiple organ failure (MOF), is closely related to severe infection. ${ }^{12-15}$ Mechanically, activation of the coagulation system and downregulation of the anticoagulant pathway result in high levels of thrombin expression and fibrin deposition in the microcirculation. ${ }^{16,17}$ Currently, dysfunction of the renal microvascular system is considered a major contributing factor to septic AKI. ${ }^{18}$ Hence, coagulation dysfunction is potentially associated with septic AKI. A considerable number of studies have evaluated the relationships between platelets and MOF in sepsis. ${ }^{19-21}$ In addition, other studies have focused on the value of several biomarkers of endothelial injury for predicting AKI in critically ill patients and not just sepsis-induced AKI. ${ }^{22-24}$ However, few studies have focused on the role of coagulative biomarkers, including APTT and D-dimer, in predicting AKI development and mortality in patients with septic shock.

Accordingly, coagulative biomarkers (eg, APTT, PT and D-dimer level) may be efficient predictors of AKI, whereas knowledge regarding the relationship between coagulopathy and sepsis-induced AKI remains limited. The present study aimed to explore the relationship between early coagulative biomarkers and the development of AKI and to measure the predictive value of these biomarkers for 30-day mortality in patients with septic shock caused by IAI.

\section{Patients and methods}

\section{Patients}

This observational retrospective study was conducted in the surgical ICU of the Second Affiliated Hospital of
Zhejiang University, a teaching hospital in Zhejiang Province in China. From January 1, 2013, to December 31, 2016, 1701 critically ill patients were admitted to the surgical ICU. Among these admissions, 138 patients who met the criteria for septic shock (The Third International Consensus Definitions for Sepsis and Septic Shock (Sepsis-3)) were enrolled (Figure 1). The following exclusion criteria were applied: (1) age younger than 18 years old; (2) ICU stay of less than $24 \mathrm{hrs;} \mathrm{(3)} \mathrm{repeated} \mathrm{admis-}$ sion to the same ICU; (4) no available coagulative measurements; (5) criteria for septic shock (Sepsis-3) not met within $24 \mathrm{hrs}$ after admission to the ICU; (6) chronic renal failure (CKD), acute or chronic liver failure (ALF or CLF), or a known hematologic disease affecting the coagulation cascade; (7) use of anticoagulation therapy to treat such disorders as deep venous thrombosis (DVT), coronary atherosclerotic heart disease or myocardial infarction (MI); or (8) septic shock caused by an infection outside the abdomen. All patients in the study were followed for more than 30 days.

IAI was defined as an infection that is limited to a hollow viscus or extends into a normally sterile area of the abdomen, such as the peritoneal cavity, mesentery, retroperitoneum, other abdominal organ, or the abdominal wall. $^{25,26}$

We defined patients with AKI according to the criteria of the Kidney Disease Improving Global Outcomes (KDIGO) classification during the ICU stay. ${ }^{11}$ The lowest $\mathrm{SCr}$ recorded one year previously in the hospital records was considered the baseline, as it provided a reasonable reflection of the patients' premorbid baseline. If the patient did not have a baseline SCr on record, baseline SCr was estimated using the equation from the Modification of Diet in Renal Disease (MDRD) Study, which assumes that the baseline eGFR is $75 \mathrm{~mL} / \mathrm{min}$ per $1.73 \mathrm{~m}^{2}$, according to the KDIGO guideline. ${ }^{11,27}$

\section{Data collection}

We collected patient data from electronic and paper medical records and recorded them on a previously designed esheet with five parts: (1) demographic data: age and sex; (2) admission status data: the source of IAI, comorbidities (hypertension) and Charlson Comorbidity Index (CCI); (3) admission laboratory data: serum procalcitonin (PCT), serum N-Terminal pro-brain natriuretic peptide (NTproBNP), serum creatinine ( $\mathrm{SCr}$ ), serum lactate (Lac), activated partial thromboplastin time (APTT), prothrombin time (PT), international normalized ratio (INR), plasma 


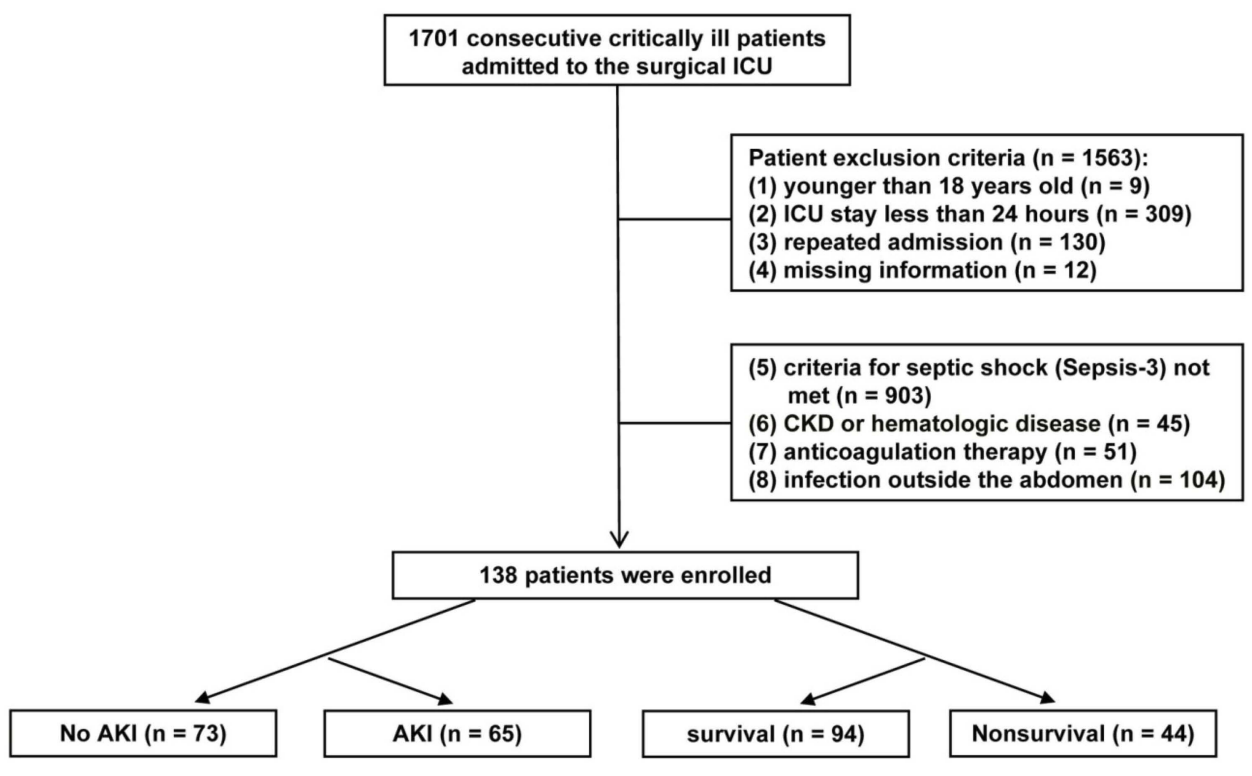

Figure I Flowchart of the enrolled patients.

fibrinogen (Fg), D-dimer and platelets (PLT); (4) data from the first $24 \mathrm{hrs}$ in the ICU: Sequential Organ Failure Assessment (SOFA) score, Acute Physiology and Chronic Health Evaluation (APACHE) II score and cumulative fluid balance (calculated using all recorded fluid input and output); and (5) primary outcomes: maximum AKI stage during ICU stay, 30-day mortality, use of renal replacement therapy (RRT), ventilation duration and length of ICU stay.

\section{Statistics}

All the data were divided into two categories: (1) continuous variables and (2) categorical data. The measurement data were expressed as the mean (standard deviation (SD)) or median (interquartile range (IQR)). Categorical data were expressed as the number (percentage). The data were compared using odds ratios (ORs) within 95\% confidence intervals (CIs) in the univariate analysis. After adjustment for model 1 or model 2, multivariable logistic regression (stepwise procedure) was used to analyze the relationship between coagulative biomarkers and the development of AKI. Model 1 included age, sex, hypertension, CCI, PCT, NT-proBNP, Lac and SOFA score. Model 2 included age, sex, hypertension, CCI, PCT, NT-proBNP, Lac and APACHE II score. Receiver operating characteristic (ROC) curve analysis was used to evaluate the value of coagulative biomarkers for predicting the development of AKI. Additionally, Cox regression (stepwise procedure) was used to identify predictors of 30-day mortality. All data were analyzed with SPSS software (version 19.0, Chicago, IL, USA) and GraphPad Prism software (version 5.01, San Diego, CA, USA).

\section{Results}

\section{Patients' baseline characteristics}

The baseline data are shown in Table 1 and indicate that the disorder most prone to IAI was gastroduodenal disease (42\%), including gastroduodenal perforation, intestinal necrosis and Crohn's disease, followed by biliary disease (22\%). The median SOFA and APACHE II scores reached 10.0 (IQR, 8.0-12.0) and 19.5 (IQR, 14.0-25.0), respectively. The incidence of AKI was 47\%, and stage 2 AKI (21\%) accounted for nearly half of that percentage. The 30 -day mortality rate was $44 \%$.

\section{Relationship between coagulative biomarkers on admission to the ICU and AKI}

The univariable cohort analysis is shown in Table 2. The patients who developed AKI exhibited a dramatically higher SOFA score, APACHE II score and mortality rate. PCT differed significantly between the AKI group and non-AKI group. The parameters of organ dysfunction, including NT-proBNP, SCr and Lac, were significantly higher in both the AKI group and nonsurvivor group. The cumulative fluid balance in the first $24 \mathrm{hrs}$ and the 
Table I Baseline characteristics

\begin{tabular}{|c|c|}
\hline Parameters & All $(n=138)$ \\
\hline \multicolumn{2}{|l|}{ Demographic data } \\
\hline Age (y), median (IQR) & $69.0(57.0-80.0)$ \\
\hline Male sex, n (\%) & $85(62)$ \\
\hline \multicolumn{2}{|l|}{ Admission status data } \\
\hline \multicolumn{2}{|l|}{ Source of intra-abdominal infection } \\
\hline Gastroduodenal disease, $\mathrm{n}(\%)$ & $58(42)$ \\
\hline Biliary disease, $\mathrm{n}(\%)$ & $30(22)$ \\
\hline Colorectal disease, $\mathrm{n}(\%)$ & $21(15)$ \\
\hline Ileocecal and appendiceal disease, $\mathrm{n}(\%)$ & $15(11)$ \\
\hline Abdominal abscess, n (\%) & $9(7)$ \\
\hline Severe acute pancreatitis, n (\%) & $5(4)$ \\
\hline \multicolumn{2}{|l|}{ Comorbidities } \\
\hline Hypertension, n (\%) & $55(40)$ \\
\hline $\mathrm{CCl}$, median (IQR) & $1.0(0.0-2.0)$ \\
\hline Data for the first 24 hrs in the ICU & \\
\hline SOFA score, median (IQR) & $10.0(8.0-12.0)$ \\
\hline APACHE II score, median (IQR) & $19.5(14.0-25.0)$ \\
\hline $\begin{array}{l}\text { Cumulative fluid balance }(\mathrm{mL}) \text {, median } \\
\text { (IQR) }\end{array}$ & $\begin{array}{l}5827.5(4795.0- \\
7220.0)\end{array}$ \\
\hline \multicolumn{2}{|l|}{ Primary outcomes } \\
\hline No AKI, n (\%) & $73(53)$ \\
\hline \multicolumn{2}{|l|}{ Maximum AKI stage during ICU stay, n (\%) } \\
\hline Stage I & $18(13)$ \\
\hline Stage 2 & $29(2 I)$ \\
\hline Stage 3 & $18(13)$ \\
\hline Death within 30 days, $\mathrm{n}$ (\%) & $44(32)$ \\
\hline RRT, n (\%) & $31(22)$ \\
\hline Ventilation duration (days), median (IQR) & $4.4(2.0-10.0)$ \\
\hline Length of ICU stay (days), median (IQR) & $6.1(2.9-13.7)$ \\
\hline
\end{tabular}

Abbreviations: $\mathrm{CCl}$, Charlson Comorbidity Index; SOFA score, Sequential Organ Failure Assessment score; APACHE II score, Acute Physiology and Chronic Health Evaluation II score; AKI, acute kidney injury; RRT, renal replacement therapy; ICU, intensive care unit.

use of RRT during the ICU stay were remarkably increased according to the severity of disease.

After adjustment for age, sex, hypertension, CCI, PCT, NT-proBNP, Lac and the SOFA score in model 1, APTT $(\mathrm{OR}=1.074, \quad 95 \% \quad$ CI $1.030-1.120, \quad p=0.001), \quad \mathrm{PT}$ $(\mathrm{OR}=1.162, \quad 95 \% \quad \mathrm{CI} \quad 1.037-1.302, \quad p=0.010), \quad \mathrm{INR}$ $(\mathrm{OR}=3.923,95 \%$ CI 1.322-11.640, $p=0.014)$ and $\mathrm{D}-$ dimer level $(\mathrm{OR}=1.098,95 \%$ CI 1.002-1.202, $p=0.045)$ on admission to the ICU were independently associated with AKI (Table 3). After adjustment for age, sex, hypertension, CCI, PCT, NT-proBNP, Lac and the APACHE II score in model 2, APTT $(\mathrm{OR}=1.074,95 \%$ CI 1.032-1.118, $p<0.001)$, PT $(\mathrm{OR}=1.168,95 \%$ CI 1.052-1.298, $p=0.004)$ and D-dimer level $(\mathrm{OR}=1.096,95 \%$ CI 1.003-1.197, $p=0.042$ ) on admission to the ICU were significantly related to AKI. In both models, we found that APTT, PT and D-dimer level on admission to the ICU were significant risk factors for AKI.

The ability of coagulative biomarkers to predict the development of AKI was evaluated using the ROC curve. The area under the curve (AUC) values of APTT, PT, D-dimer level and INR were 0.857 (0.797-0.918), $0.832(0.765-0.898), 0.844(0.780-0.909)$ and 0.828 (0.761-0.895), respectively, after adjustment for model 1 (Figure 2A). The AUC values of APTT, PT and D-dimer level were 0.848 (0.784-0.912), 0.826 (0.757-0.894) and 0.820 (0.749-0.892), respectively, after adjustment for model 2 (Figure 2B, Table 4).

\section{Relationship between APTT and 30-day mortality}

The filtered variables were further evaluated by Cox regression analysis (Table 5). The results showed that APTT was an independent predictive factor of 30-day mortality. The adjusted OR of model 1 was $1.065(1.025-1.107, p=0.001)$ and that of model 2 was $1.061(1.023-1.102, p=0.002)$. PLT had statistical significance only after adjustment for model $2(\mathrm{OR}=0.990,95 \%$ CI $0.981-0.998, p=0.016)$. The patients were categorized into a High APTT group and a Low APTT group based on the median APTT (46.8 s). Kaplan-Meier curve analysis indicated that the High APTT group had a significantly higher 30-day mortality than the Low APTT group (Figure 3A). Moreover, the patients were categorized into four groups based on the interquartile range of plasma APTT levels (Q1, APTT $\leq 39.8 \mathrm{~s}$; Q2, $39.8 \mathrm{~s}<$ APTT $\leq 46.8 \mathrm{~s}$; Q3, $46.8 \mathrm{~s}<$ APTT $\leq 53.3 \mathrm{~s}$; Q4, APTT $>53.3 \mathrm{~s}$ ). The 30-day mortality rates were $20 \%$ in Q1, 24\% in Q2, $31 \%$ in Q3 and 53\% in Q4. Histogram analysis indicated that the longer the plasma APTT was, the higher the risk of 30-day mortality (Figure 3B).

\section{Discussion}

In the present study, the activation of the coagulation system was observed in both the AKI and nonsurvivor groups. Using multivariable logistic regression analysis, we found that APTT, PT and D-dimer level on admission to the ICU were significant risk factors for AKI. Moreover, multivariable Cox regression analysis showed that 


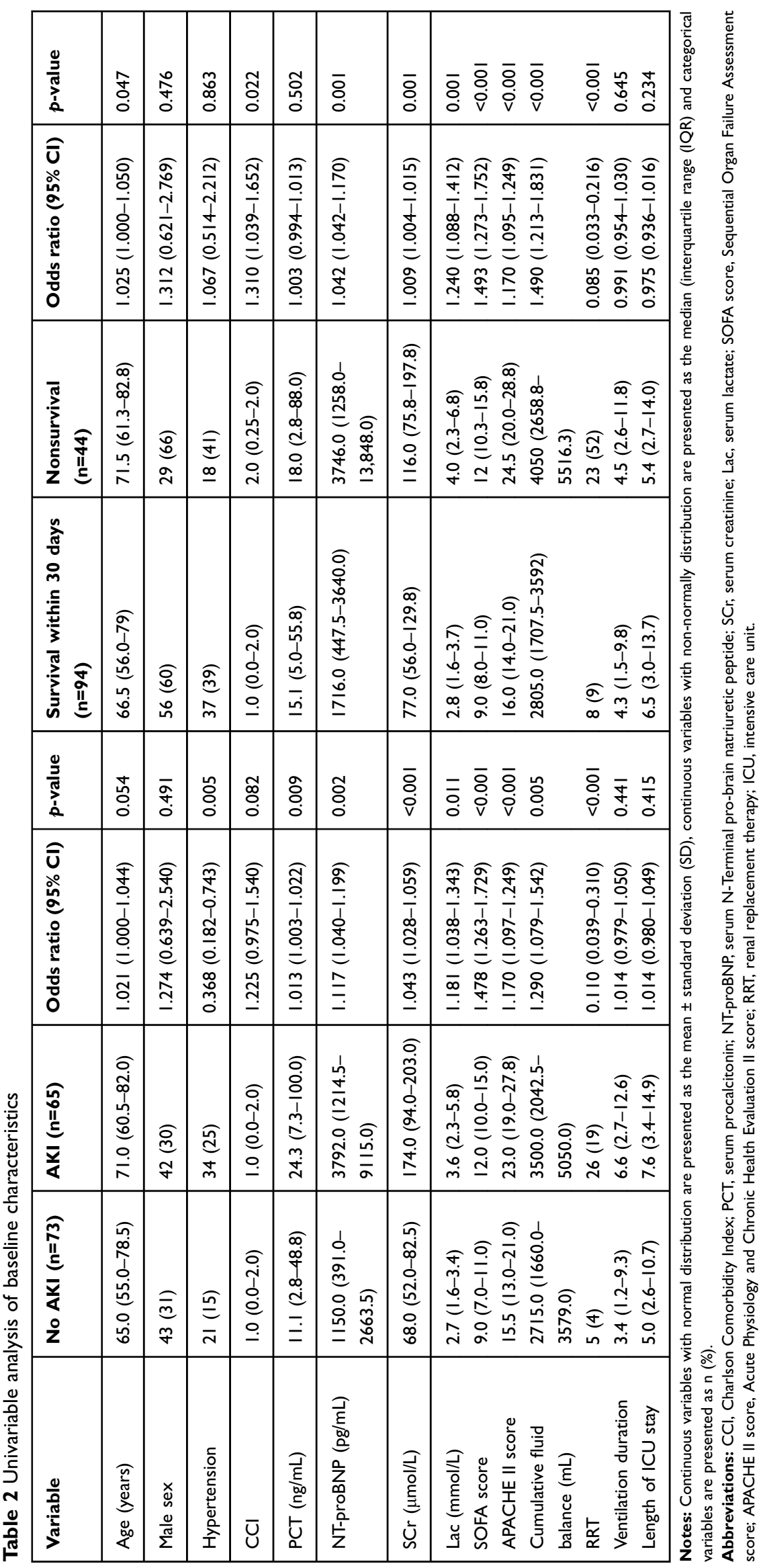




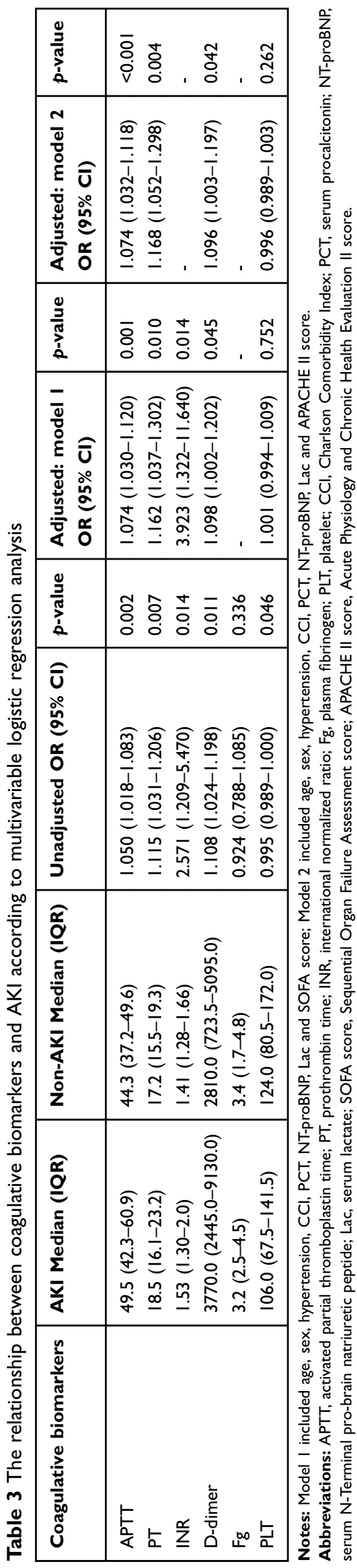

prolonged APTT was independently associated with high mortality in patients with septic shock caused by IAI.

Previous evidence has shown that platelet activation plays an important role in sepsis and is associated with MOF. ${ }^{19,21}$ Thrombocytopenia has been associated not only with coagulation mediators but also with bone marrow suppression in sepsis. ${ }^{19,28}$ Whether coagulative biomarkers can predict the development of septic AKI remains unclear. Recently, Katayama et al studied the interactive connection between sepsis-induced AKI and several biomarkers of endothelial injury and the coagulation system. ${ }^{24}$ The authors used univariable analysis to show that PT and PLT were associated with the development of AKI in patients with sepsis, which was consistent with our findings. However, APTT and D-dimer level were not included among the biomarkers of the coagulation system in their study. SAC correlates not only with the development of AKI but also with an increased risk of mortality. ${ }^{29}$ Benediktsson et al showed that prolonged APTT and PT at the time of ICU admission was associated with increased mortality in sepsis patients, without evaluating the relationship between D-dimer level and mortality. ${ }^{30}$ Compared with the existing literature, the current data further concluded that PT, APTT and D-dimer level were significantly associated with the development of AKI in patients with septic shock. Moreover, our finding showed that APTT on admission to the ICU was an independent predictor of the exacerbation of septic AKI and provides further evidence of the usefulness of APTT as a biomarker of mortality.

Severe infection induces and aggravates endothelial injury and initiates the intrinsic coagulation pathway through the consumption of coagulation factors. Lipopolysaccharide (LPS) or bacteria can directly trigger the upregulation of tissue factor (TF) in circulating monocytes, which aggravates the endothelial injury of vascular organs and comes into contact with blood when the endothelium is injured. ${ }^{31}$ The extrinsic coagulation pathway, considered as the initiator of the coagulation system, is triggered by the TF/factor VIIa complex, which plays a pivotal role in the initiation of thrombin generation. Due to the destruction of endothelial integrity, FXIIa can initiate the intrinsic pathway of thrombin generation by activation of coagulation factor XI. ${ }^{16,32}$ Furthermore, cross-talk and feedback occur between the extrinsic pathway and intrinsic pathway. Sepsis-induced activation of the coagulation system is initiated by the extrinsic pathway and amplified by the intrinsic pathway via the cross-talk and feedback loop. ${ }^{33} \mathrm{PT}$ and APTT are used to evaluate the extrinsic and 
A

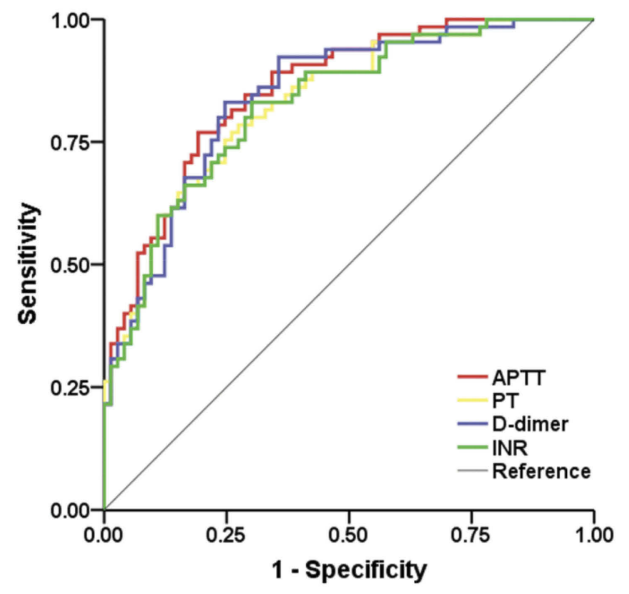

B

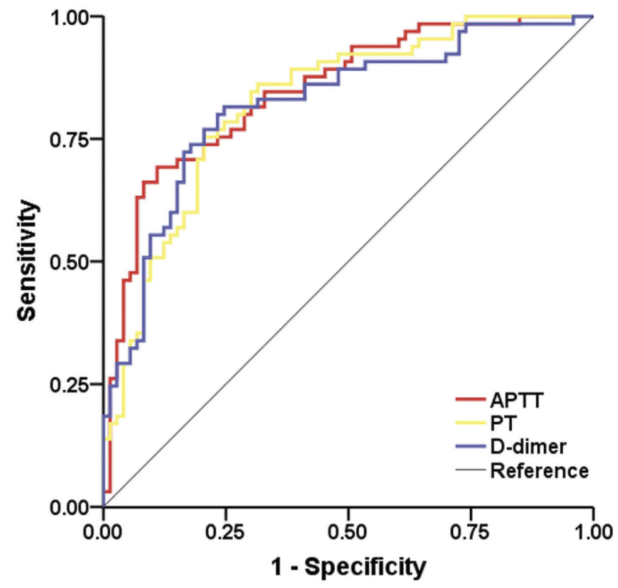

Figure 2 Comparison of the area under the curve (AUC) of coagulative biomarkers for predicting AKI. (A) Comparison of the AUC for APTT, PT, INR and D-dimer level after adjustment for model I. (B) Comparison of the AUC for APTT, PT and D-dimer level after adjustment for model 2.

Table 4 The AUC of coagulative biomarkers for predicting AKI after adjustment for baseline characteristics in model I or model 2

\begin{tabular}{|l|l|l|l|l|l|}
\hline Coagulative biomarkers & AUC & $\mathbf{9 5 \%} \mathbf{C l}$ & Sensitivity (\%) & Specificity (\%) & Youden Index \\
\hline Adjusted: model I & & & & & \\
APTT & 0.857 & $0.797-0.918$ & 76.9 & 80.8 & 0.577 \\
PT & 0.832 & $0.765-0.898$ & 78.5 & 72.6 & 0.511 \\
D-dimer & 0.844 & $0.780-0.909$ & 83.1 & 75.3 & 0.584 \\
INR & 0.828 & $0.761-0.895$ & 83.1 & 69.9 & 0.530 \\
\hline Adjusted: model 2 & & & & & \\
APTT & 0.848 & $0.784-0.912$ & 69.2 & 79.0 & 0.582 \\
PT & 0.826 & $0.757-0.894$ & 75.4 & 79.5 & 0.549 \\
D-dimer & 0.820 & $0.749-0.892$ & 81.5 & 75.3 & 0.568 \\
\hline
\end{tabular}

Notes: Model I included age, sex, hypertension, CCI, PCT, NT-proBNP, Lac and SOFA score; Model 2 included age, sex, hypertension, CCI, PCT, NT-proBNP, Lac and APACHE II score.

Abbreviations: APTT, activated partial thromboplastin time; PT, prothrombin time; INR, international normalized ratio; CCI, Charlson Comorbidity Index; PCT, serum procalcitonin; NT-proBNP, serum N-Terminal pro-brain natriuretic peptide; Lac, serum lactate; SOFA score, Sequential Organ Failure Assessment score; APACHE II score, Acute Physiology and Chronic Health Evaluation II score.

intrinsic coagulation pathways, respectively. The present study indicated that SAC is hypocoagulable based on the prolonged PT and APTT on ICU admission in patients with septic AKI. PT, APTT and D-dimer level may be the most commonly measured biomarkers of coagulation in the clinic. However, the role of coagulopathy in sepsisinduced AKI remains poor owing to the lack of detailed information from biopsies or autopsies. Additional large studies still need to be conducted to explore the pathophysiology and improve the prognosis in patients with septic AKI.

Our findings have several implications for clinicians. First, for patients with septic shock, early monitoring and intervention are crucially important. Therefore, we should strengthen the monitoring of renal function to prevent the development of septic AKI among patients who have prolonged APTT and PT and an increased D-dimer level at the time of ICU admission. Second, patients with SAC accompanied by AKI can receive RRT to contribute to the recovery of renal function and reduce mortality. ${ }^{34,35}$ With effective intervention and treatment, we can slow the deterioration of renal function, shorten the stay in the ICU and even reduce the waste of medical resources. Moreover, clinicians may minimize the use of drugs that impair renal function when the patient appears to be in a state of hypocoagulability. Third, whether all septic patients should receive systemic anticoagulation and when to provide anticoagulation therapy are under 


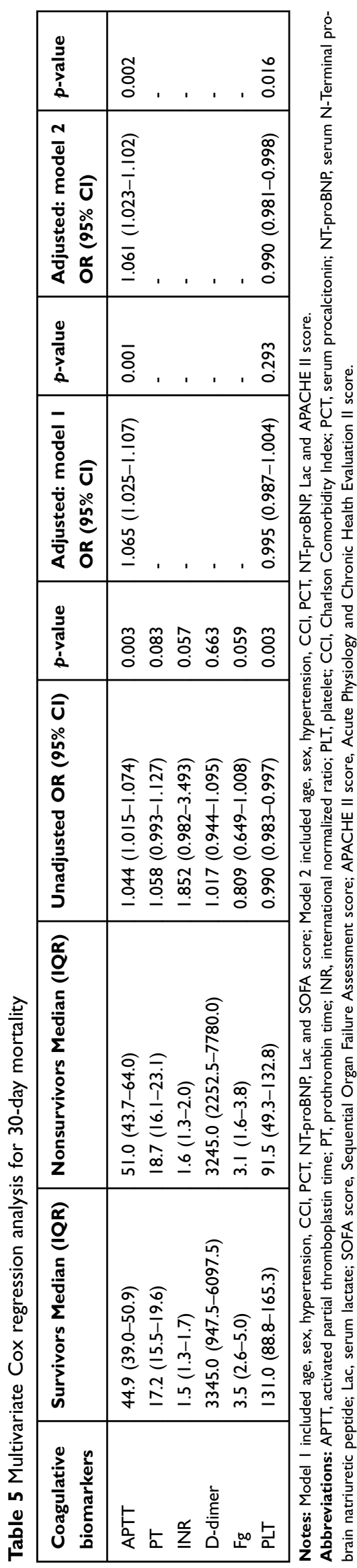

debate. ${ }^{36,37}$ Recently, some studies ${ }^{38,39}$ have indicated that heparin therapy was associated with decreased mortality in patients with sepsis. In contrast, some researchers ${ }^{37,40}$ have recommended not administering anticoagulation therapy for all patients with sepsis because of the increased risk of bleeding. At minimum, the study provides laboratory evidence that patients in the septic AKI group or nonsurvivor group were hypocoagulable during the early stage. These patients may have an increased risk of bleeding if given anticoagulation therapy.

This research also has several strengths. Above all, to the best of our knowledge, this study is the first to assess the role of APTT and D-dimer level in the development of AKI in patients with septic shock and to explore the relationship between APTT and mortality. Additionally, the eligibility criteria for our study were rigorous in the exclusion of patients with atherosclerosis or DVT to reduce selection bias.

It is important to recognize limitations. First, this was a single-center retrospective study. Although the sample was relatively small, the study has important positive results and clinical significance. The results should be verified with a large study in the future. Second, not all the patients had baseline information available. Some patients' baseline creatinine levels could be estimated using the MDRD formula according to the KDIGO guidelines. However, it eliminated information bias in the research. Third, at present, global coagulation assays such as thrombelastography (TEG) and clot waveform analysis have several advantages compared with traditional coagulation tests. ${ }^{41}$ It is unfortunate that TEG parameters were not tested in every patient because of their time and effort requirements. Therefore, more research is needed to explore the potential relationship among global coagulation assays, organ failure and mortality in critically ill patients. Despite these limitations, we believe that our findings are important and should prompt further research to verify our results.

\section{Conclusion}

The present study provides new insights into patients with AKI caused by IAI. By analyzing the patient data, our study indicated that APTT, PT and D-dimer level on admission to the ICU were significant predictors of AKI. Furthermore, APTT on admission to the ICU was an independent predictor of 30-day mortality. 
A

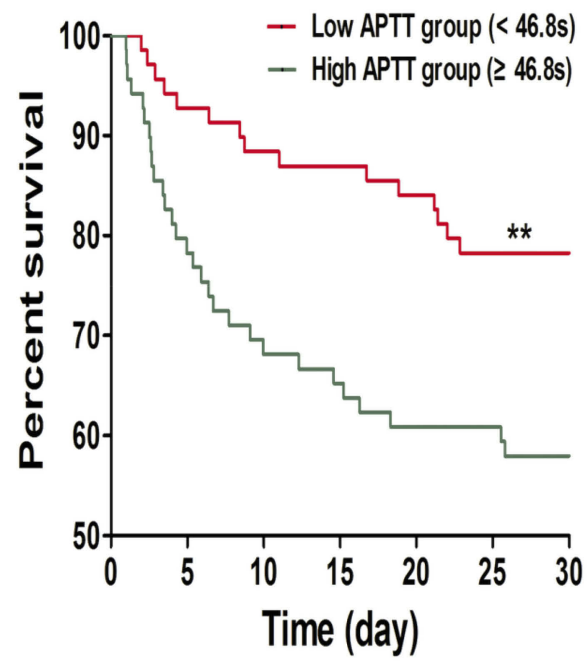

B

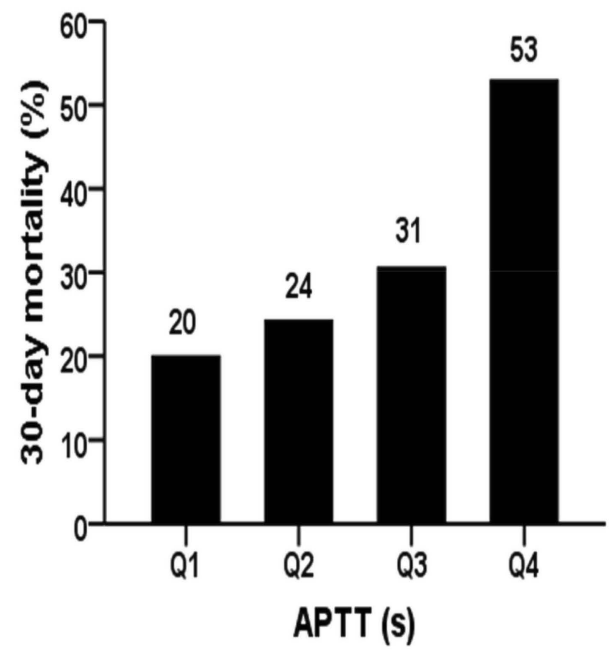

Figure 3 (A) Kaplan-Meier survival curves of 30-day mortality according to plasma APTT levels. (B) The 30-day mortality rates according to the interquartile range of plasma APTT levels. (QI, APTT $\leq 39.8 \mathrm{~s}$; Q2, $39.8 \mathrm{~s}<$ APTT $\leq 46.8 \mathrm{~s} ;$ Q3, $46.8 \mathrm{~s}<$ APTT $\leq 53.3 \mathrm{~s}$; Q4, APTT >53.3 s).

\section{Ethical consideration}

This study was conducted in accordance with the Declaration of Helsinki and was approved by the Ethical Committee of The Second Affiliated Hospital, School of Medicine, Zhejiang University. All patient data were analyzed in anonymity. Patient consent was waived by the ethics committee, as no individual data were published, nor was any intervention was performed on patients.

\section{Acknowledgment}

We appreciate the nurses and physicians in the surgical ICU who assisted with the study.

\section{Disclosure}

The authors report no conflicts of interest in this work.

\section{References}

1. Singer M, Deutschman CS, Seymour CW, et al. The third international consensus definitions for sepsis and septic shock (Sepsis-3). JAMA. 2016;315(8):801-810. doi:10.1001/jama.2016.0287

2. Vincent JL, De Backer D. Circulatory shock. N Engl J Med. 2013;369 (18):1726-1734. doi:10.1056/NEJMra1208943

3. Levy MM, Rhodes A, Phillips GS, et al. Surviving Sepsis Campaign: association between performance metrics and outcomes in a 7.5-year study. Crit Care Med. 2015;43(1):3-12. doi:10.1097/CCM.0000000 000000723

4. Angus DC, van der Poll T. Severe sepsis and septic shock. $N$ Engl $J$ Med. 2013;369(9):840-851. doi:10.1056/NEJMra1208623

5. Boldingh QJ, de Vries FE, Boermeester MA. Abdominal sepsis. Curr Opin Crit Care. 2017;23(2):159-166. doi:10.1097/MCC.0000000 000000388
6. Hoste EA, Bagshaw SM, Bellomo R, et al. Epidemiology of acute kidney injury in critically ill patients: the multinational AKI-EPI study. Intensive Care Med. 2015;41(8):1411-1423. doi:10.1007/s00 134-015-3934-7

7. Chawla LS, Eggers PW, Star RA, Kimmel PL. Acute kidney injury and chronic kidney disease as interconnected syndromes. $N$ Engl $J$ Med. 2014;371(1):58-66. doi:10.1056/NEJMra1214243

8. Gordon AC, Mason AJ, Thirunavukkarasu N, et al. Effect of early vasopressin vs norepinephrine on kidney failure in patients with septic shock: the VANISH randomized clinical trial. JAMA. 2016;316(5):509-518. doi:10.1001/jama.2016.10485

9. Vanmassenhove J, Kielstein J, Jorres A, Biesen WV. Management of patients at risk of acute kidney injury. Lancet. 2017;389 (10084):2139-2151. doi:10.1016/S0140-6736(17)31329-6

10. Bellomo R, Kellum JA, Ronco C, et al. Acute kidney injury in sepsis. Intensive Care Med. 2017;43(6):816-828. doi:10.1007/s00134-0174755-7

11. Kellum JA, Lameire N, Aspelin P, et al. Kidney disease: improving global outcomes (KDIGO) acute kidney injury work group. KDIGO clinical practice guideline for acute kidney injury. Kidney Inter Suppl. 2012;2:1-138.

12. Gando S. Microvascular thrombosis and multiple organ dysfunction syndrome. Crit Care Med. 2010;38(2 Suppl):S35-S42. doi:10.1097/ CCM.0b013e3181c9e31d

13. Perrin J, Charron C, Francois JH, et al. Coagulation phenotypes in septic shock as evaluated by calibrated automated thrombography. Shock. 2015;43(1):74-79. doi:10.1097/SHK.0000000000000271

14. Thiery-Antier N, Binquet C, Vinault S, Meziani F, Boisramé-Helms $\mathrm{J}$, Quenot J-P. Is thrombocytopenia an early prognostic marker in septic shock? Crit Care Med. 2016;44(4):764-772. doi:10.1097/ CCM.0000000000001520

15. Levi M, van der Poll T. Coagulation and sepsis. Thromb Res. 2017;149:38-44. doi:10.1016/j.thromres.2016.11.007

16. Levi M, van der Poll T. Inflammation and coagulation. Crit Care Med. 2010;38(2 Suppl):S26-S34. doi:10.1097/CCM.0b013e3181c $98 \mathrm{~d} 21$

17. Fourrier F. Severe sepsis, coagulation, and fibrinolysis: dead end or one way? Crit Care Med. 2012;40(9):2704-2708. doi:10.1097/ CCM.0b013e318258ff30 
18. Verma SK, Molitoris BA. Renal endothelial injury and microvascular dysfunction in acute kidney injury. Semin Nephrol. 2015;35(1):96107. doi:10.1016/j.semnephrol.2015.01.010

19. de Stoppelaar SF, van 't Veer C, van der Poll T. The role of platelets in sepsis. Thromb Haemost. 2014;112(4):666-677. doi:10.1160/TH14-020126

20. Mihajlovic D, Lendak D, Mitic G, et al. Prognostic value of hemostasis-related parameters for prediction of organ dysfunction and mortality in sepsis. Turk $J$ Med Sci. 2015;45(1):93-98.

21. Greco E, Lupia E, Bosco O, Vizio B, Montrucchio G. Platelets and multi-organ failure in sepsis. Int J Mol Sci. 2017;18(10):2200. doi:10.3390/ijms18102200

22. Su CM, Cheng HH, Hung $\mathrm{CW}$, et al. The value of serial serum cell adhesion molecules in predicting acute kidney injury after severe sepsis in adults. Clin Chim Acta. 2016;457:86-91. doi:10.1016/j.cca.2016. 04.008

23. Liu KL, Lee KT, Chang CH, Chen YC, Lin SM, Chu PH. Elevated plasma thrombomodulin and angiopoietin-2 predict the development of acute kidney injury in patients with acute myocardial infarction. Critical Care. 2014;18(3):R100. doi:10.1186/cc13712

24. Katayama S, Nunomiya S, Koyama K, et al. Markers of acute kidney injury in patients with sepsis: the role of soluble thrombomodulin. Critical Care. 2017;21(1):229. doi:10.1186/s13054-017-1686-1

25. Solomkin JS, Mazuski JE, Bradley JS, et al. Diagnosis and management of complicated intra-abdominal infection in adults and children: guidelines by the Surgical Infection Society and the Infectious Diseases Society of America. Surg Infect. 2010;11(1):79-109. doi:10.1089/sur.2009.9930

26. Mazuski JE, Tessier JM, May AK, et al. The surgical infection society revised guidelines on the management of intra-abdominal infection. Surg Infect. 2017;18(1):1-76. doi:10.1089/sur.2016.261

27. Kellum JA, Lameire N. Diagnosis, evaluation, and management of acute kidney injury: a KDIGO summary (Part 1). Critical Care. 2013;17(1):204. doi:10.1186/cc12734

28. Hartwig J, Italiano J Jr. The birth of the platelet. J Thromb Haemost. 2003;1(7):1580-1586.

29. Lyons PG, Micek ST, Hampton N, Kollef MH. Sepsis-associated coagulopathy severity predicts hospital mortality. Crit Care Med. 2018;46:736-742. doi:10.1097/CCM.0000000000002997

30. Benediktsson S, Frigyesi A, Kander T. Routine coagulation tests on ICU admission are associated with mortality in sepsis: an observational study. Acta Anaesthesiol Scand. 2017;61(7):790-796. doi:10.1111/aas.12918
31. Pernerstorfer T, Hollenstein U, Hansen JB, et al. Lepirudin blunts endotoxin-induced coagulation activation. Blood. 2000;95(5):17291734.

32. Puy C, Ngo ATP, Pang J, et al. Endothelial PAI-1 (Plasminogen Activator Inhibitor-1) blocks the intrinsic pathway of coagulation, inducing the clearance and degradation of FXIa (Activated Factor XI). Arterioscler Thromb Vasc Biol. 2019;39(7):1390-1401. doi:10.1161/ATVBAHA.119.312619

33. Aird WC. Vascular bed-specific hemostasis: role of endothelium in sepsis pathogenesis. Crit Care Med. 2001;29(7 Suppl):S28-S35. doi:10.1097/00003246-200107001-00013

34. Zarbock A, Kellum JA, Schmidt C, et al. Effect of early vs delayed initiation of renal replacement therapy on mortality in critically ill patients with acute kidney injury: the ELAIN randomized clinical trial. JAMA. 2016;315(20):2190-2199. doi:10.1001/jama.2016.5828

35. Karvellas CJ, Farhat MR, Sajjad I, et al. A comparison of early versus late initiation of renal replacement therapy in critically ill patients with acute kidney injury: a systematic review and metaanalysis. Critical Care. 2011;15(1):R72. doi:10.1186/cc10324

36. Meziani F, Gando S, Vincent JL. Should all patients with sepsis receive anticoagulation? Yes. Intensive Care Med. 2017;43(3):452454. doi:10.1007/s00134-016-4621-z

37. van der Poll T, Opal SM. Should all septic patients be given systemic anticoagulation? No. Intensive Care Med. 2017;43(3):455-457. doi:10.1007/s00134-016-4607-x

38. Zarychanski R, Abou-Setta AM, Kanji S, et al. The efficacy and safety of heparin in patients with sepsis: a systematic review and metaanalysis. Crit Care Med. 2015;43(3):511-518. doi:10.1097/ CCM.0000000000000763

39. Wang C, Chi C, Guo L, et al. Heparin therapy reduces 28-day mortality in adult severe sepsis patients: a systematic review and meta-analysis. Critical Care. 2014;18(5):563. doi:10.1186/cc13712

40. Warren BL, Eid A, Singer P, et al. Caring for the critically ill patient. High-dose antithrombin III in severe sepsis: a randomized controlled trial. JAMA. 2001;286(15):1869-1878. doi:10.1001/jama.286.15.1869

41. Wikkelso A, Wetterslev J, Moller AM, Afshari A. Thromboelastography (TEG) or thromboelastometry (ROTEM) to monitor haemostatic treatment versus usual care in adults or children with bleeding. Cochrane Database Syst Rev. 2016;8(8):CD007871.
Infection and Drug Resistance

\section{Publish your work in this journal}

Infection and Drug Resistance is an international, peer-reviewed openaccess journal that focuses on the optimal treatment of infection (bacterial, fungal and viral) and the development and institution of preventive strategies to minimize the development and spread of resistance. The journal is specifically concerned with the epidemiology of antibiotic resistance and the mechanisms of resistance development and diffusion in both hospitals and the community. The manuscript management system is completely online and includes a very quick and fair peerreview system, which is all easy to use. Visit http://www.dovepress.com/ testimonials.php to read real quotes from published authors. 